\title{
O PAPEL DO ENFERMEIRO NO ACOMPANHAMENTO DE PRÉ NATAL DE BAIXO RISCO UTILIZANDO A ABORDAGEM CENTRADA NA PESSOA - GESTANTE
}

\author{
Rachel Sarmento Reis ${ }^{1}$, Chennyfer Dobbins Abi Rached ${ }^{2}$
}

1 Especialista em Saúde da Família - Faculdade Unyleya - . São Paulo - Brasil - Email: São Paulo - Brasil

2 Doutora em Saúde Coletiva; Mestre em Economia da Saúde pela Universidade Federal de São Paulo (UNIFESP); Docente do Programa de Mestrado Profissional em Gestão em Sistemas de Saúde - Universidade Nove de Julho - UNINOVE. São Paulo - Brasil email: chennyferr@yahoo.com.br ORCID: https://orcid.org/0000-0002-4499-3716

\section{RESUMO}

Trata-se de uma revisão narrativa sobre a importância da consulta do enfermeiro durante o pré-natal da gestante de baixo risco, utilizando a abordagem centrada na pessoa - gestante. O pré-natal é o acompanhamento da evolução da gestação que visa cuidar da saúde da mulher e do seu bebê até que o parto ocorra, também é o momento que a gestante vivencia diferentes sentimentos, por isso o estabelecimento de relação com a enfermagem se faz imprescindível. O pré-natal quando realizado com qualidade desempenha importante papel na redução da mortalidade materna e infantil. Informações sobre as diferentes vivências devem ser trocadas entre as mulheres e os profissionais de saúde. A consulta de enfermagem é uma atividade que irá proporcionar ao enfermeiro (a) condições para atuar de forma direta e independente com a paciente, caracterizando dessa forma sua autonomia. Segundo a Secretaria Municipal de Saúde do Rio de Janeiro, a abordagem centrada na pessoa - gestane deve ser uma ferramenta primordial na Estratégia de Saúde da Familia, pois propicia o vínculo, atendimento integral e fortalecimento da longitudinalidade do cuidado alem de ser uma tecnologia leve, utilizada pelo profissional que permite idenificar as múltiplas vulnerabilidades, fazendo com que diminuam se as chances de complicações e mortalidade materna e infantil e uma boa qualidade do pré-natal. Espera-se que este estudo contribua para reflexão do enfermeiro, quanto a sua importância nesse contexto, visando uma assistência à gestante cada vez mais humanizada e científica.

Palavras Chaves: Enfermeiro. Pré-natal. Baixo risco. Gestante. Humanização. 


\begin{abstract}
This is a narrative review about the importance of the nurse consultation during the prenatal care of the low-risk pregnant woman using the person-centered approach. The prenatal care is the follow-up of the evolution of gestation that aims to take care of the health of the woman and her baby until the childbirth occurs, it is also the moment that the pregnant woman experiences different feelings, so the establishment of relationship with the nursing becomes indispensable. Prenatal care when performed with quality plays an important role in reducing maternal and infant mortality. Information on the different experiences should be exchanged between women and health professionals. The nursing consultation is an activity that will provide nurses with the conditions to act directly and independently with the patient, characterizing their autonomy. According to the Municipal Health Department of Rio de Janeiro, the person - centered approach should be a key tool in the Family Health Strategy, since it provides the link, comprehensive care and strengthening of care longitudinality, besides being a light technology, used by the professional that allows identifying the multiple vulnerabilities, causing them to decrease if the chances of complications and maternal and infant mortality and a good prenatal quality. It is hoped that this study contributes to the nurses' reflection, as to their importance in this context, aiming at assisting the increasingly humanized and scientific pregnant women.
\end{abstract}

KEY WORDS: Nurse. Antenatal visit. Low risk. Pregnant. Humanization. 


\section{INTRODUÇÃO}

O pré-natal foi instituído no início do século XX no mundo, chegando ao Brasil entre as décadas de 20 e 30 . Tendo nessa época enfoque apenas na mulher, em diminuir os agravos para sua saúde, sem pensar no binômio gestante e feto. Entre as décadas de 50 e 60 , com a diminuição das taxas de morte materna, começou a se pensar no feto. Assim, com os avanços tecnológicos e sociais, o pré-natal constituiu-se e se firmou, transformando-se na prática assistencialista que acontece hoje.

Segundo Osava \& Tanaka (1997), em termos históricos, a enfermagem sempre esteve presente no acompanhamento e avaliação de mulheres em período gestacional, visto que a enfermeira exerce papel fundamental na realização de parto e vem recebendo várias designações no decorrer dos anos como parteira, obstetriz e enfermeira obstetra.

O caráter preventivo do pré-natal é fundamental para diminuir os índices de mortalidade materna e perinatal, pois um acompanhamento durante o período gestacional bem feito previne patologias, tais como anemias, doenças hipertensiva gestacional (pré-eclâmpsia, eclâmpsia); também favorece o preparo psicológico para o parto, além de garantir a perfeita estruturação do organismo fetal, prevenção do abortamento e o risco de parto prematuro e óbito perinatal dentre outras vantagens que trazem ganhos a saúde dessa mulher de maneira única, como empoderamento de si, cuidados a sua saúde e praticas de prevenção e promoção mesmo após o termino da gestação e puerperio. Constituindo assim um dos grandes objetivos e desafios com que o profissional em Saúde da Família precisa lidar em sua prática diária.

De acordo com Neme (2000), é nesse cenário que o profissional enfermeiro tem um estreito contato com as gestantes e suas preocupações no período gestacional. E ainda, o Ministério da Saúde (Texto 9 - Assistência pré-natal do Manual Técnico) alerta que: “...a adesão das mulheres ao pré-natal está relacionado com a qualidade da assistência prestada pelos serviços e pelos profissionais de saúde, o que, em última análise, será essencial para a redução dos elevados índices de mortalidade materna e perinatal, verificada no Brasil”. (BRASIL, 2000).

\subsection{Problema de Pesquisa}

Frente à expansão da Atenção Primária em nosso país e a atuação do enfermeiro no pré-natal de baixo risco na Estratégia em Saúde da Família, verifica-se a necessidade de mais pesquisas de enfermagem em como fazê-lo com qualidade: (i) para um acompanhamento que visa o binômio mãe/bebê; (ii) a redução de mortalidade materna e fetal; (iii) e a classificação de risco gestacional em todas as consultas, diante de tantas 
responsabilidades que tal profissional tem sobre si. E ainda, como fazê-lo de forma com que o enfermeiro consiga utilizar técnicas para favorecer o cuidado integral a essa mulher, que nesse momento está gestante, mas que depois continuará sendo assistida pelo mesmo, no cuidado longitudinal realizado com ela e com sua família, inclusive com o bebê gestado. Desta forma, quais as ferramentas podemos utilizar para favorecer a construção desse vínculo e genuinamente o cuidado integral?

\subsection{Justificativa}

Apesar de termos vários artigos, manuais do Ministério da Saúde, protocolos de enfermagem e protocolos municipais, pouco se discute sobre como alcançar a gestante de forma a entender e reconhecer todos os riscos que a mesma possui e como fazê-la reconhecê-los, ou como a própria equipe possa reconhecer. A diversidade do perfil dessas usuárias é bastante abrangente. Ainda é comum observarmos, na prática, a dificuldade de tais profissionais no cuidado a gestante. Por isto questiona-se: Qual o papel da enfermagem e suas intervenções para o atendimento qualificado a gestante? Quais os desafios para comunicação e cuidado integral com elas?

$\mathrm{O}$ enfermeiro faz parte de uma equipe multidisciplinar e possui atribuições importantes para o processo de acolhimento e sequencia no atendimento desta mulher, principalmente no contexto da atenção básica de saúde, por ter atribuições específicas. Sendo uma delas, a realização da Consulta de Enfermagem, com avaliação integral da mulher.

Tal profissional pode através de sua formação, que é voltada para o cuidado, ser um facilitador durante o pré-natal. Conduzindo a gestante a empoderar-se de si, e ser a protagonista da gestação. Além de aumentar a autonomia dessa mulher através do cuidado, e tudo isso através da ferramenta de escuta sensível e do cuidado centrado na pessoa, centrado na gestante, centrado na mulher.

O papel do enfermeiro como cuidador é buscar integralmente a saúde destas mulheres, seja prescrevendo cuidados de enfermagem e medicamentos previstos em programas de saúde e protocolos das instituições de saúde, mantendo esquemas terapêuticos, solicitando exames complementares e fortalecendo o vínculo entre a gestante e sua equipe.

Durante os últimos oito anos, com a grande expansão em Saúde da Família no Município do Rio de Janeiro, e atuando como enfermeira de equipe, percebo como que o cuidado centrado na pessoa pode fazer com que consigamos alcançar toda a integralidade do sujeito. E como isso traz um grande potencial no cuidado ao outro, em 
especial as gestantes e suas famílias. Percebo efeitos e avanços significativos no cuidado integral a gestante, ao preparo da mesma para chegada de seu bebê e sua família ou rede de apoio. E após o parto, sobre todo seguimento dessa puérpera, que logo depois, será apenas a mulher assistida longitudinalmente. $\mathrm{O}$ vínculo aumentado devido ao maior número de consultas a essa mulher, utilizando a abordagem centrada nela faria com que toda a assistência durante o pré-natal, e após o mesmo, tornasse ouro significado com um cuidado integral e humanizado como proposto na Estratégia em Saúde da Família.

Nesta perspectiva, pretende-se contribuir para análise e discussão sobre como o enfermeiro, durante o acompanhamento do pré-natal, pode através da abordagem centrada na pessoa - gestante, realizar um acompanhamento integral. E por meio dele, a identificação de riscos e vulnerabilidades que podem fazer com que essa gestante tenha maior possibilidade de mortalidade. Além disso, demonstrar a possibilidade de um fortalecimento do vínculo que se manterá com o profissional e a mulher após o término do pré-natal, facilitando que seja seguida a longitudinalidade do cuidado próprio em saúde da família.

\subsection{Objetivos}

\subsubsection{Objetivo geral}

Propor que a abordagem centrada na pessoa - gestante, possa ser uma ferramenta utilizada no acompanhamento de pré-natal de baixo risco, realizado pelo enfermeiro em Saúde da Família.

\subsubsection{Objetivos específicos}

A - Descrever o pré-natal de baixo risco, realizado pela Estratégia de saúde da família; B - Apresentar a abordagem centrada na pessoa - gestante; C - Relatar o papel do enfermeiro no acompanhamento de pré-natal de baixo risco, utilizando a abordagem centrada na pessoa, na Estratégia de saúde da família.

\subsection{Metodologia}

Neste estudo adotou-se como estratégia metodológica a pesquisa bibliográfica, um estudo exploratório de abordagem qualitativa, que permite uma melhor abrangência da realidade profissional. 
Segundo Minayo (2004), a preocupação com os significados tem ganhado força. O como na pesquisa avaliamos o significado do papel do enfermeiro para colaborar com o empoderamento da gestante durante o pré-natal, ressaltando questões que até recentemente passavam desapercebidas. Entre elas, dar autonomia a gestante durante o pré-natal, visto que a mesma é quem recebe todas as alterações fisiológicas desencadeadas durante a gestação e futuramente ao ato de partejar.

Deste modo, na elaboração deste trabalho foi realizada uma pesquisa na literatura nacional sobre o tema proposto, utilizando como fontes textos acadêmicos, livros, monografias, dissertações e teses. Além de protocolos e manuais técnicos do Ministério da Saúde e da Secretaria Municipal de Saúde do Rio de Janeiro.

Reafirmando a importância deste tipo de estudo, Tretini e Paim (1999, p. 68) asseguram que "a seleção criteriosa de uma revisão de literatura pertinente ao problema significa familiarizar-se com textos e, por eles, reconhecer os autores e o que eles estudaram anteriormente sobre o problema a ser estudado".

\section{REVISÃO DE LITERATURA}

\subsection{CAPÍTULO 1 - O acompanhamento de pré-natal de baixo risco na Estratégia de Saúde da Família (ESF)}

O Pré-natal foi instituído no início do século XX no mundo, chegando ao Brasil entre as décadas de 20 e 30. Tendo nessa época enfoque apenas na mulher, em diminuir os agravos para sua saúde, sem pensar no binômio gestante e feto. Nos anos 50 e 60, com a diminuição das taxas de morte materna, começou a se pensar no feto. Assim, com os avanços tecnológicos e sociais, o pré-natal constituiu-se e se firmou, transformandose na prática assistencialista que acontece hoje.

Nos dias de hoje, o Pré-natal consiste em um acompanhamento da evolução da gestação, que visa cuidar da saúde da mulher e de seu bebê até que o parto ocorra. Mas, ele vai muito além do cuidar da saúde física, pois durante o mesmo se orienta a mulher sobre sua gravidez, os cuidados que ela deve ter neste período, a nutrição, os exercícios, o trabalho de parto, o parto, o aleitamento e outros temas. (AQUINO, 2005).

Ele é o início, meio e fim da preparação da mulher para todo o período gestacional, pois são, nas consultas, que a gestante irá conhecer e aprender como cuidarse e prevenir-se, bem como, receber orientações de como lidar com os diversos fatores que influenciam na gestação e já introduzir como ela poderá cuidar de seu bebê, quando ele nascer. 
Conforme Brasil (2006), o Ministério da Saúde alerta que para essa atenção ser de qualidade e humanizada, deve-se incorporar condutas acolhedoras, sem intervenções desnecessárias e que haja fácil acesso a serviços de saúde de qualidade, com ações que integrem todos os níveis de atenção: promoção, prevenção e assistência à saúde da gestante e do recém nascido.

A assistência ao pré-natal tem como objetivo o acolhimento da gestante desde o diagnóstico da gestação, visando acolher a mulher em um momento de transição e modificações físicas e emocionais de forma individualizada (BRASIL, 2006).

Para o Ministério da Saúde (2006), o acolhimento é um aspecto essencial da política de humanização, resulta na recepção da mulher, desde sua chegada à unidade básica, onde os profissionais de saúde se responsabilizam por ela, ouvindo suas queixas e permitindo que ela expresse suas preocupações, angustias, garantindo atenção primordial e articulando com outros serviços de saúde, fornecendo dessa maneira continuidade a assistência, quando necessário

Para muitas mulheres, o momento da gestação é um período que vai lhes trazer muitas perguntas e dúvidas, o enfermeiro ou outro profissional que a acompanhe, deve ter condições e conhecimentos necessários para esclarecer, no que forem possíveis, os questionamentos de cada paciente, dando-lhes o apoio necessário e fornecendo orientações que influenciem numa gravidez longe de riscos que possam ser prejudiciais à mãe e ao bebê.

Segundo Rios e Vieira (2007) o período do Pré-natal é uma época de preparação física e psicológica para o parto e para maternidade e como tal, é um momento de intenso aprendizado e uma oportunidade para os profissionais da equipe de saúde desenvolver a educação como dimensão do processo de cuidar.

O medo do desconhecido e os cuidados a serem prestados ao recém-nascido são alguns fatores de tensão para a gestante, e é da competência do acompanhamento de pré-natal saber lidar, para dissipar toda e qualquer dúvida a esse respeito durante todo o acompanhamento dessa mulher (Sshnnyder, 2014).

Segundo Bezerra (2009) em nosso país, a qualidade do acompanhamento de Pré Natal continua sendo um ponto crítico da assistência à saúde da mulher, e ao binômia mãe-bebê. Mesmo com a melhoria dos indicadores, ainda e notável a taxa de mortalidade materna e fetal.

A morte materna tem sido um grande desafio à saúde pública brasileira, afinal ela é um dos grandes indicadores de saúde feminina. A Organização Mundial da Saúde (OMS) define como morte materna a que ocorre durante a gestação ou dentro de um período de 42 dias após o término desta, independente da duração ou da localização da 
gravidez, devido a qualquer causa relacionada com a gravidez ou por medidas relacionadas a ela. (BRASIL, 2007)

As causas de morte materna são divididas em dois grupos: mortes obstétricas diretas e mortes obstétricas indiretas. As mortes obstétricas diretas ocorrem devido complicações obstétricas durante a gravidez, parto ou puerpério, durante o período de acompanhamento do pré-natal, causadas por intervenções, omissões ou tratamento incorreto. Já as mortes obstétricas indiretas são causadas por doenças já existentes anteriormente à gestação, ou que são desenvolvidas neste período e se agravam pelos efeitos fisiológicos da gestação (RIQUINHO, 2006).

Segundo Riquinho (2006), no Brasil possuímos elevadas taxas de mortalidade materna. E, tem sido um grande desafio para os serviços de saúde, e para a sociedade; configurando-se como um grande problema de saúde pública que atinge de forma desigual as classes sociais menos favorecidas.

Nesse contexto reafirma-se mais uma vez a grande potência que o pré-natal é, pois é uma das principais formas de prevenção de morte materna e fetal por meio da atenção pré-natal, disponibilizado pela rede pública de saúde, na ESF, gratuito, de fácil acesso, e de direito de todas as gestantes (BRASIL, 2012).

Nogueira e Oliveira (2017) definem o pré-natal da seguinte forma:

Compreende-se por pré-natal um dos mais completos conjuntos de procedimentos clínicos e educativos, oferecidos a um grupo populacional específico, que tem o objetivo de promover a saúde e identificar precocemente problemas que possam trazer riscos para a saúde da gestante e do concepto, além de diagnóstico e tratamento adequado dos problemas que possam vir a ocorrer nesse período, com amplo potencial de impacto sobre a morbimortalidade materno- infantil.

Por esse motivo o pré-natal deve ter início precoce, cobertura universal, ser realizado de forma periódica, estar integrado com as demais ações preventivas e curativas; deve ser respeitado um número mínimo de consultas e seu sucesso depende, em grande parte, do momento em que ele se inicia (NOGUEIRA e OLIVEIRA, 2017)

O principal objetivo da atenção pré- natal e puerperal é acolher a mulher desde o início da gravidez, assegurando, ao fim da gestação, o nascimento de uma criança saudável e a garantia do bem-estar materno e neonatal, contribuindo com a redução da mortalidade materna e infantil. Uma atenção pré-natal e puerperal qualificada se dá através da incorporação de condutas acolhedoras e sem intervenções desnecessárias; do fácil acesso a serviços de saúde de qualidade, com ações que integrem todos os níveis da atenção: promoção, prevenção e assistência à saúde da gestante e do recém-nascido, 
desde o atendimento ambulatorial básico ao atendimento hospitalar para alto risco.(RIO DE JANEIRO, 2016)

A fim de reverter essa situação, iniciativas foram implementadas pelo setor saúde e pelas entidades de classe, cabendo destacar o incentivo à participação dos profissionais de enfermagem obstétrica no acompanhamento ao pré-natal e parto de gestantes de baixo risco obstétrico. (Bezerra, 2009)

O objetivo do acompanhamento pré-natal é assegurar o desenvolvimento da gestação, permitindo o parto de um recém-nascido saudável, sem impacto para a saúde materna, inclusive abordando aspectos psicossociais e as atividades educativas e preventivas. (BRASIL, 2012)

A realização do pré-natal representa papel fundamental em termos de prevenção e/ou detecção precoce de patologias, tanto maternas, como fetais. Permitindo um desenvolvimento saudável do bebê e reduzindo os riscos da gestante. Informações sobre as diferentes vivências devem ser trocadas entre as mulheres e os profissionais de saúde. Essa possibilidade de intercâmbio de experiências e conhecimentos é considerada a melhor forma de promover a compreensão do processo de gestação. (BRASIL, 2000)

Para Nogueira e Oliveira (2017) a assistência pré-natal é uma ferramenta imprescindível para que o ciclo gravídico-puerperal ocorra de maneira mais segura, o que contribui de modo eficaz para a redução das mortes maternas. Ressaltando que a assistência pré-natal depende de procedimentos relativamente simples, podendo ser conduzido em sua plenitude na atenção básica em saúde, pois não carece de alta tecnologia e pode contemplar as necessidades das gestantes na maioria das vezes.

De acordo com Brasil (2012) é necessário 10 Passos para o Pré-Natal de Qualidade na Atenção Básica:

$1^{\circ}$ PASSO: Iniciar o pré-natal na Atenção Primária à Saúde até a $12^{\mathrm{a}}$ semana de gestação (captação precoce);

$2^{\circ}$ PASSO: Garantir os recursos humanos, físicos, materiais e técnicos necessários à atenção pré-natal;

$3^{\circ}$ PASSO: Toda gestante deve ter assegurado a solicitação, realização e avaliação em termo oportuno do resultado dos exames preconizados no atendimento pré-natal;

$4^{\circ}$ PASSO: Promover a escuta ativa da gestante e de seus(suas) acompanhantes, considerando aspectos intelectuais, emocionais, sociais e culturais e não somente um cuidado biológico: "rodas de gestantes";

$5^{\circ}$ PASSO: Garantir o transporte público gratuito da gestante para o atendimento prénatal, quando necessário; 
$6^{\circ}$ PASSO: É direito do(a) parceiro(a) ser cuidado (realização de consultas, exames e ter acesso a informações) antes, durante e depois da gestação: "pré-natal do(a) parceiro(a)";

$7^{\circ}$ PASSO: Garantir o acesso à unidade de referência especializada, caso seja necessário;

$8^{\circ}$ PASSO: Estimular e informar sobre os benefícios do parto fisiológico, incluindo a elaboração do "Plano de Parto";

9० PASSO: Toda gestante tem direito de conhecer e visitar previamente o serviço de saúde no qual irá dar à luz (vinculação);

10 PASSO: As mulheres devem conhecer e exercer os direitos garantidos por lei no período gravídico-puerperal.

Magalhães e Moreira in Corrêa et al. (2004) afirmam que o sucesso do acompanhamento pré-natal depende ainda de alguns fatores decisivos como: o interesse e participação da gestante e de seus familiares; que estes reconheçam a importância deste acompanhamento; que o prénatalista seja competente para identificar e corrigir as alterações na evolução da gravidez, dedicado e solícito atendendo corretamente a gestante e humano para acolher e motivar a gestante a prosseguir o pré-natal seguindo as orientações propostas.

Em 2006 o Brasil adotou a Estratégia em Saúde da Família (ESF) como modelo assistencial para reorganizar a atenção primária á saúde. Países com uma potente orientação para ESF apresentam melhores condições de saúde, custos mais baixos e maior satisfação das pessoas.

A Estratégia de saúde da família (ESF), é uma estratégia de reorganizaçãoda Atenção primária a saúde. No Brasil, a ESF iniciou se através do Programa de Agesntes Comunitários de Saúde (PACS) em 1991, como parte do processo de reforma do setor da saúde e que, visava aumentar o acesso ao sistema de saúde e incrementar as ações de prevenção e promoção de saúde (BRASIL, 2007).

Porém em 2006, o Programa de Saúde da família (PSF), passou a ser implementada através da portaria $\mathrm{N}^{\circ} 648$, de 28 de março de 2006 , como prioridade para reorganizar a Atenção básica que tem como seus fundamentos o acesso universal e contínuoaos serviços de saúde de qualidade, reafirmando os outros princípios básicos do SUS: equidade, descentralização, integralidade e participação da comunidade, mediante o cadastramento e a vinculação dos usuários (BRASIL, 2004)

O princ ipal obejetivo da estratégia de saúde da família, é a transformação do modelo tradicional de assistência no qual, entre outras práticas, os trabalhadores da saúde aguardavam passivamente á procura dos serviços pela população. Os serviços de 
saúde tem o papel dinâmico de ir ao encontro da população, principalmente por meio da visita domiciliar, cujo o foco é centrado na família e nos indivíduos. O formato de saúde que milita, que busca o usuário de forma ativa, dentro do que chama-se "lei dos cuidados inversos", que é aq busca do usuário que não vem ativamen te a unidade ou na sua equipe (BRASIL, 2007)

A unidade básica de saúde (UBS/ESF) deve ser a porta de entrada preferencial da gestante no sistema de saúde. É o ponto de atenção estratégico para melhor acolher suas necessidades, inclusive proporcionando um acompanhamento longitudinal e continuado, principalmente durante a gravidez (BRASIL, 2012)

Neste contexto, as equipes de atenção básica devem se responsabilizar pela população de sua área de abrangência, mantendo a coordenação do cuidado mesmo quando a referida população necessita de atenção em outros serviços do sistema de saúde (BRASIL, 2012).

O Programa de Humanização no Pré-natal e Nascimento (PHPN) foi instituído pelo Ministério da Saúde (MS) através da Portaria/GM n.569, em 01 de junho de 2000, visando concentrar esforços no sentido de reduzir as altas taxas de morbimortalidade materna e perinatal; adotar medidas que assegurem a melhoria do acesso, da cobertura e da qualidade do acompanhamento pré-natal, da assistência ao parto, puerpério e neonatal; e ampliar as ações já adotadas pelo MS na área de atenção à gestante, como os investimentos nas redes estaduais de assistência à gestação de alto risco (BAULI, 2010).

A grande pretensão era que a Política de Humanização fosse um marcador de águas anunciando o paradigma da humanização como novo modelo de atenção á mulher durante a gestação e o parto e o cuidado centrado na pessoa da gestante.

Apesar de todos os esforços para melhorar a saúde da mulher, ainda havia necessidade de fortalecer a assistência prestada e, então, no ano de 2004, o Ministério da Saúde elaborou a Política Nacional de Atenção Integral a Saúde da Mulher princípios e diretrizes (PNAISM). Esta política foi criada em parceria com diversos setores da sociedade, como o movimento das mulheres, o movimento negro, o das trabalhadoras rurais, a sociedade cientifica, as organizações não governamentais, os gestores do SUS e as agências de cooperação internacional. (BRASIL, 2009).

A PNAISM reforça a humanização da assistência à mulher, levando em conta que qualidade e humanização são indissociáveis e que humanizar não é só dar um tratamento digno a mulher, mas que também se trata de acessibilidade a todos os níveis de atenção a saúde e direito a referência e contra referência. A partir disso, a mulher poderá então ser vista na sua integralidade, como um sujeito autônomo e participativo 
nesse processo. Com isso, haverá garantias de atendimento a suas necessidades e consequentemente melhorando a qualidade da sua assistência (BRASIL, 2009).

A expansão da ESF e a implementação do PHPN e PNAISM são estratégias empregadas para a redução da mortalidade materna no Brasil, e assistência integral á mulher e seu bebê durante o período gestacional.

O Ministério da Saúde no ano de 2000, implementou algumas ações de dependem exclusivamente da atuação da ESF como na execução e acompanhamento do Pré - Natal de baixo risco, tais como :

- Identificar as gestantes precocemente para que iniciem o cuidado no primeiro trimestre gestacional;

- Busca ativa das gestantes faltosas;

- Ter acesso ao, no mínimo, 6 consultas de Pré-natal;

- Acesso a consulta puerperal;

- Desenvolver ações educativas com as gestantes e assim melhorar a qualidade da assistência ao Pré-natal de baixo risco.

A consulta de Pré-natal deve ser realizada de maneira integral, visando um atendimento de qualidade, não se detendo apenas ao mínimo de consultas realizadas, mas a toda abordagem da gestante de acordo com todas as vulnerabilidades apresentadas por ela.

O Pré-Natal de baixo risco, segundo Brasil (2012) pode ser todo acompanhado na Atenção primária pela equipe de saúde da Família. Risco é a probabilidade e não certeza; não são apenas as doenças ou condições biológicas adversas que aumentam a chance de a gestante ter um desenlace gestacional infeliz. (NETTO, 2005).

A presença de fatores protetores na gestante, como, por exemplo, a boa nutrição, a imunização completa e as adequadas condições higiênico-sanitárias, favorece a condição de saúde e diminui a probabilidade de danos a ela. (NETTO, 2005).

Nos casos em que não há necessidade de se utilizar alta densidade tecnológica em saúde e nos quais a morbidade e a mortalidade materna e perinatal são iguais ou menores do que as da população em geral, as gestações podem ser consideradas como de baixo risco. Assim definida, a gravidez de baixo risco somente pode ser confirmada ao final do processo gestacional, após o parto e o puerpério. O processo dinâmico e a complexidade das alterações funcionais e anatômicas que ocorrem no ciclo gestacional exigem avaliações continuadas e específicas em cada período (BRASIL, 2012)

Nos grupos de baixo risco, as necessidades são resolvidas por procedimentos simples, que constituem o chamado primeiro nível de assistência. Já o atendimento da 
gestante de alto risco acontece em um segundo ou terceiro nível de assistência, dotado de pessoal, técnicas e equipamentos adequados à solução de cada caso. (NETTO, 2005).

$\mathrm{Na}$ primeira consulta é realizada a anamnese que aborda desde aspectos epidemiológicos até a situação da gravidez atual, seguido do exame físico que deve ser geral e específico (gineco-obstétrico) e solicitando exames complementares para um atendimento amplo e completo durante o pré-natal. É realizado o cálculo da idade gestacional, o qual estima a idade do feto/ tempo de gravidez, e também a data provável do parto, realiza a avaliação sobre o estado nutricional da gestante, por meio do índice de massa corporal (IMC), inicia-se o controle da pressão arterial. Nas consultas seguintes deve-se realizar a revisão da ficha pré-natal, anamnese atual sucinta e verificação do calendário de vacinação, realizando o controle materno e fetal, também calcular a idade gestacional, o IMC, controle da pressão arterial, palpação obstétrica e medida uterina, ausculta dos batimentos cardíacos fetais, registro de movimentos fetais, detecção de edema e interpretação dos resultados (BRASIL, 2006).

Durante as consultas de pré-natal, o enfermeiro é responsável por realizar ações educativas para a gestante e sua família, acompanhar gestações de baixo risco, solicitar exames de rotina e orientar tratamento de acordo com o protocolo da instituição, e também coletar exame citopatológico. Foi conferido também ao enfermeiro, declarar os nascidos vivos dos partos realizados em instituições de saúde e domicílios, desde que estejam devidamente cadastrados pelas Secretarias Municipais de Saúde (SMS) como profissionais responsáveis por estes atendimentos (BRASIL, 2012).

\subsection{CAPÍTULO 2 - A abordagem centrada na pessoa - gestante}

A Abordagem Centrada na Pessoa foi desenvolvida por Carl R. Rogers, renomado psicólogo norte-americano do século XX. Ele foi o primeiro a exercer a função de psicoterapeuta e o pioneiro no interesse pela pesquisa científica em psicoterapia. Como integrante do movimento humanista, contribuiu com a consolidação dos princípios da Psicologia Humanista. Seu trabalho com grupo centrado em mediação de conflitos e a crença de que esse trabalho estaria ajudando a sociedade na busca pela paz fez com que ele tivesse seu nome indicado para o prêmio Nobel da Paz no ano de 1987 (MOIRA, 2007).

Através dessa abordagem psicológica, Rogers construiu seu diferencial de psicoterapia sustentado na existência de uma tendência individual para o crescimento e saúde, na ênfase dos elementos emocionais em detrimento dos intelectuais, na priorização do presente em detrimento do passado e no reconhecimento do papel da 
relação terapêutica na experiência de crescimento. Em defesa da ideia de que a personalidade humana tende a saúde e ao bem-estar, Rogers desenvolveu atitudes facilitadoras e recursos interventivos que permitem o resgate do potencial realizador existente em todo ser humano. Esse potencial é reconhecido como pilar da teoria, denominado Tendência Atualizante e associado a base motivacional da vida (WOOD e outros, 2008).

Posteriormente, Rogers identificou que esta compreensão não se aplicava apenas ao atendimento psicológico, mas a todas as relações humanas . Independente da situação é possível estabelecer uma relação aceitadora e permissiva, na qual haja o interesse genuíno pelo outro. Deste modo, as condições básicas para o desenvolvimento interpessoal, passaram a representar um jeito de ser e de estar nas relações (WOOD e outros, 2008).

O cuidado efetivo à gestante exige prestar atenção às experiências em relação à saúde, a todas as expectativas que a mulher traz em si no período gestacional, o apoio de seu companheiro ou da sua família, o seu estilo de vida, dentre tantos outros fatores e elementos que devem ser "coletados" ao iniciar um acompanhamento de Pré-natal. Mas, o mais importante a todas as coisas referidas é necessário estar atento ao centro do cuidado: a gestante.

Este processo de repensar a prática dos profissionais da saúde vai além da adaptação teórico/prática. Relaciona-se diretamente com as relações interpessoais que estes profissionais estabelecem. O projeto do SUS alcança a individualidade, a particularidade das pessoas em seu contexto e, para que o cuidado à saúde se aproxime da compreensão das singularidades de cada pessoa, os profissionais precisam desenvolver habilidades relacionais que os aproximem da compreensão e do cuidado integral das pessoas (BRASIL, 2006)

O profissional de saúde além das ações e procedimentos técnicos ligados à sua área específica, estabelece sempre, com as pessoas que atende, relações interpessoais. Seu trabalho depende, portanto, da qualidade técnica e da qualidade interacional. (...) a abordagem da qualidade interacional também torna necessário o estudo de vários temas teóricos e a reflexão sobre o desenvolvimento de atitudes (MOIRA, 2007)

Com o objetivo de que o atendimento à saúde chegasse a toda a população, incluiu-se nas diretrizes de saúde pública ações e serviços de promoção, prevenção, reabilitação e tratamento da saúde. Este movimento se desenvolve através de três níveis de atenção: a básica, a média e a alta complexidade, também denominadas atenções primária, secundária e terciária (BRASIL, 2006). 
Com a abrangência destas três atenções, E buscou-se trazer a integralidade das ações para o atendimento, considerando a saúde em sua totalidade. Essas ações estão voltadas para os cidadãos, tanto na sua individualidade, quanto na coletividade. A atenção básica pode ser considerada a porta de entrada do atendimento em saúde por possuir menor complexidade. Inicialmente, o cidadão seria atendido através da atenção básica representada principalmente por postos e centros de saúde. Nos casos em que há necessidade de recursos mais complexos, a pessoa seria encaminhada para os outros serviços da rede de maior complexidade .Tem como estratégia principal o Programa de Saúde da Família e, assim como o SUS, em geral, preconiza o acesso universal e contínuo da população aos serviços de saúde (BRASIL 2007).

O modelo biomédico, aplicado durante muitos anos tinha seu foco apenas no corpo do indivíduo, era apenas baseado em uma base conceitual para entender as dimensões biológicas do adoecer, ou de estar "saudável", reduzindo a pessoa a sua doença. O foco é no corpo, não na pessoa (CERON, 2012).

Nesse modelo, não se tinha espaço para a pessoa. Como vimos no capítulo acima na Estratégia em Saúde da Família, modelo vigente, a dinâmica do cuidado é totalmente diferente leva-se em conta todo cuidado proposto através da humanização, cuidado integral, vínculo. E isso nos conota que a ESF, vai totalmente ao encontro da abordagem centrada na pessoa, ao contrário do modelo biomédico (BRASIL, 2006).

Ao aproximar as habilidades relacionais, das técnicas, conjuntamente com vínculo podemos aproximar sobre a convergência entre as políticas públicas de saúde no Brasil e a proposta da Abordagem Centrada na Pessoa (ACP). Esta se trata de uma proposta psicológica que se aproxima teoricamente das diretrizes propostas pelas políticas públicas nos serviços de saúde do Brasil, que se alinha ao princípios e diretrizes do SUS e de toda a abordagem realizada Estratégia de saúde da família (CERON, 2012).

Uma perspectiva que integra os profissionais da saúde para abordar a pessoa na sua integralidade deve contemplar o desenvolvimento de uma postura que considere a realidade da pessoa como algo dinâmico, singular, relacional e que considere o humano existente em cada indivíduo. Esse é o único jeito de promover um verdadeiro processo de humanização e isso porque, "a humanização dos cuidados em saúde pressupõe considerar a essência do ser, o respeito à individualidade e a necessidade de construção de um espaço concreto nas instituições de saúde que legitime o humano das pessoas envolvidas" , sejam estes os pacientes, os familiares ou os profissionais. Nessa perspectiva, passa a ser responsabilidade dos profissionais de saúde o desenvolvimento de um olhar atento e considerador, uma prática voltada para a criação de condições 
facilitadoras do desenvolvimento do protagonismo e da coresponsabilidade das pessoas na atenção à saúde, como forma de garantir a prática de alguns dos princípios norteadores da Política de Humanização (BRASIL, 2006).

Construção de autonomia e protagonismo dos sujeitos e coletivos implicados na rede do SUS; Co-responsabilidade desses sujeitos nos processos de gestão e atenção; Compromisso com a democratização das relações de trabalho e valorização dos profissionais de saúde, estimulando processos de educação permanente (BRASIL, 2007).

A Política Nacional de Atenção Básica (PNAB), sancionada através da Portaria $\mathrm{N}^{\mathrm{o}}$ 2.436, DE 21 de setembro de 2017, traz em seu texto toda a reorganização do Sistema Ùnico de Saúde, reafirmando a Estratégia em Saúde da Família como a forma de restruturar a Atenção Primária e traz em suas diretrizes o Cuidado Centrado na pessoa como descrito a seguir:

Cuidado Centrado na Pessoa: aponta para o desenvolvimento de ações de cuidado de forma singularizada, que auxilie as pessoas a desenvolverem os conhecimentos, aptidões, competências e a confiança necessária para gerir e tomar decisões embasadas sobre sua própria saúde e seu cuidado de saúde de forma mais efetiva. O cuidado é construído com as pessoas, de acordo com suas necessidades e potencialidades na busca de uma vida independente e plena. A família, a comunidade e outras formas de coletividade são elementos relevantes, muitas vezes condicionantes ou determinantes na vida das pessoas e, por consequência, no cuidado. (BRASIL, 2017)

Segundo Ceron (2012) para implementar e executar a "ferramenta" do cuidado centrado na pessoa, é necessário uma comunicação eficaz, pois é fundamental conquistar e manter vínculo com as diversas famílias do território de saúde, visando à realização do cuidado em acordo com os princípios da universalidade, da longitudinalidade e da integralidade do SUS. A ESF é a porta de entrada do sistema, e a complexidade dos casos que acessam as Equipes de Saúde da famlia, são os mais diversificados: múltiplas demandas, queixas e pedidos.

Para que a abordagem centrada na pessoa possa ser implementada de forma satisfatória é necessário construir as práticas assistenciais implementando a integralidade do atendimento, unificando o olhar sobre as mais complexas dimensões do contexto bio-psico-social familiar e comunitário, levando em todo o contexto social, ambiental, histórico e humano do território de saúde (CERON, 2012).

A abordagem centrada na pessoa é considerada uma tecnologia leve e eficaz no cuidado integral e resolutivo, assimo como o proposto na Saúde da Família. Tal abordagem possui em si alguns objetivos que se complementam aos princípios e diretrizes do SUS e da Atenção primária, segundo Ceron (2012), são eles:

- Diagnóstico precoce visando à prevenção de agravos; 
-Reconhecer situações socioambientais que comprometam a saúde;

-Estabelecimento de vínculo visando à transformação;

-Capacidade de sensibilização;

-Motivar para adesão aos cuidados à saúde;

- Educação da comunidade, fortalecendo o seu empoderamento, para a construção ativa e participativa do SUS.

As tecnologias leves se encontram em contraste com as tecnologias pesadas, tais como os equipamentos especializados, de última geração, presentes em hospitais e laboratórios especializados, especialmente no nível terciário de Atenção à Saúde. São as habilidades de comunicação verbais e não verbais, a capacidade de vínculo afetivo e as atitudes em relação aos usuários que melhoram a qualidade do atendimento e sua resolutividade (CERON, 2012).

Segundo Moira (2010) o modelo de cuidado clínica centrada na pessoa acontece quando o profissional promove uma relação de cooperação com o paciente. Nessa metodologia, os protagonistas encontram um terreno comum para abordar as preocupações do paciente, as decisões a serem tomadas e as suas ideias sobre o que ocorre e o que deve ser feito. Levam-se em conta as suas expectativas e as suas experiências pessoais e culturais em relação à gestação, e também as de sua comunidade.

Para Cerqueira (2009) as características práticas na execução da abordagem centrada na pessoa incluem algumas atitudes do profissional que direciona a consulta, tais como:

- Permitir ao paciente a livre expressão de suas preocupações mais importantes;

- Buscar que sejam verbalizadas perguntas concretas;

-Favorecer e motivar que os pacientes expliquem suas crenças e expectativas sobre a sua enfermidade;

- Facilitar a expressão emocional do paciente;

- Proporcionar informações ao paciente e esclarecer suas dúvidas;

- Envolver o paciente na construção do tratamento, buscando entrar em acordo quanto à forma como ele acontecerá. 
Segundo Ceron (2012) et al,

Uma atuação centrada na pessoa apresenta resultados positivos comparada aos modelos tradicionais de abordagem, pois: diminui a utilização dos serviços de saúde, aumenta sua satisfação, diminui queixas por má-prática, melhora a aderência aos tratamentos, reduz preocupações, melhora a saúde mental, reduz sintomas e melhora a recuperação de problemas recorrentes (CERON, 2012).

A abordagem centrada na pessoa é extremamente terapêutica, na medida que favorece a clínica ampliada, centrada na pessoa, em toda integralidade do sujeito, mas também ao que ele traz como expectativa. È uma tecnologia que permite, através da comunicação, que o usuário entenda que faz parte de todo o processo terapêutico.

Para isso, Ceron (2012) afirma algumas posturas que potencializam a Comunicação e o sucesso dessa abordagem:

- Escuta sensível, que inclusive permite o desabafo;

- Acolhimento que permite a ampliação de seu cuidado, estabelecendo o vínculo;

- Suporte que representa o continente para os sentimentos envolvidos;

- Esclarecimento que desfaz fantasias, aumenta a informação e reestrutura o pensamento, reduzindo ansiedade e depressão.

O uso de metodologias ampliadas de cuidado faz parte do esforço coletivo dos profissionais de saúde em dar mais um passo a fim de desenvolver ações de saúde de acordo com a Política Nacional de Humanização (PNH), como a construção de uma clínica ampliada e integrada, capaz de atender o usuário como sujeito, visando ao seu esclarecimento, ao desenvolvimento de sua capacidade de enfrentamento e à sua resiliência frente às situações vividas

A Política Nacional de Humanização toma o acolhimento como postura prática nas ações de atenção e gestão das unidades de saúde, o que favorece a construção de uma relação de confiança e compromisso dos usuários com as equipes e os serviços, contribuindo para a promoção da cultura de solidariedade e para a legitimação do sistema público de saúde (BRASIL, 2012).

O acolhimento da gestante por sua equipe de saúde da família, na atenção básica, implica em responsabilizar-se por toda integralidade do cuidado, desde o cadastro dessa usuária (seja na recepção da unidade, ou na visita domiciliar do agente comunitário de 
saúde) com uma escuta qualificada, sensível, para construção e favorecimento do vínculo e da avaliação de vulnerabilidades de acordo com o seu contexto social, entre outros cuidados.

Durante o seguimento dessa gestante, a abordagem centrada nela, deve garantir que ela expresse suas preocupações e suas angústias, garantindo a atenção resolutiva e a articulação com os outros serviços de saúde para a continuidade da assistência e, quando necessário, possibilitando a criação de vínculo da gestante com a equipe de saúde.

Cabe à equipe de saúde, ao entrar em contato com uma mulher gestante, esforçar se em buscar compreender os múltiplos significados da gestação para aquela mulher e sua família através da empatia - principalmente caso a gestante seja uma adolescente. A história de vida e o contexto de gestação trazidos pela mulher durante a gravidez devem ser acolhidos integralmente a partir do seu relato e de seu parceiro, ou familiares caso seja de desejo da gestante. Tal contexto implica mudanças nas relações estabelecidas entre a mulher e a família, o pai e a criança. Além disso, gera mudanças na relação da gestante consigo mesma, no modo como ela entende seu autocuidado, bem como modificações em como ela percebe as mudanças corporais, o que interfere muitas vezes no processo de amamentação.

O diálogo franco, a sensibilidade e a capacidade de percepção de quem acompanha o pré- -natal são condições básicas para que o saber em saúde seja colocado à disposição da mulher e da sua família( os atores principais da gestação) - e nesse contexto a abordagem centrada na gestante confirma o uso de essa estratégia tão eficaz.

Uma escuta aberta, sem julgamentos nem preconceitos, de forma que permita à mulher falar de sua intimidade com segurança, fortalece a gestante no seu caminho até o parto e ajuda a construir o seu conhecimento sobre si mesma, contribuindo para que tanto o parto quanto o nascimento sejam tranqüilos e saudáveis.

A escuta á uma gestante é algo mobilizador. Escutar é um ato de autoconhecimento e reflexão contínua sobre as próprias fantasias, medos, emoções, amores e desamores. Escutar é desprendimento de si. Na escuta, o sujeito dispõe-se a conhecer aquilo que talvez esteja muito distante de sua experiência de vida, o que, por isso, exige grande esforço para ele compreender e ser capaz de oferecer ajuda, ou melhor, trocar experiências, é a ferramenta, a tecnologia essencial para colocar a gestante ao centro e não o saber profissional.

Segundo o Ministério da Saúde (2012), no caderno 32, sobre a Atenção ao prénatal de baixo risco, o cuidado á gestante se expressa na relação estabelecida entre o profissional de saúde e á gestante, mediante atitudes profissionais humanizadoras, que compreendem iniciativas tais como as de: 
1. Se apresentar;

2. Chamar a usuária pelo nome;

3. Prestar informações sobre condutas e procedimentos que devam ser realizados;

4. Escutar e valorizar o que é dito por ela;

5. Garantir a privacidade e a confidencialidade das informações;

6. Incentivar a presença do(a) acompanhante (de sua escolha), entre outras iniciativas semelhantes.

O acolhimento, da gestante e sua família, a abordagem de coloca-la no cen'tro do cuidado é portanto,uma ação que pressupõe a mudança da relação profissional/ usuário(a). Como citado no início desse capítulo ao comparar o modelo Biomédico que vem sendo substituído pela ESF. Colocar a usuária, gestante como centro do cuidado utilizando esse tipo de abordagem, não é um espaço ou um local, mas uma postura ética e solidária. Portanto, ele não se constitui como uma etapa do processo, mas como ação que deve ocorrer em todos os locais e momentos da atenção à saúde, do cuidado durante o pré-natal e todo o seguimento desta usuária e sua família, durante a longitudinalidade do cuidado que é de inteira responsabilidade da ESF como coordenadora do cuidado de todas as gestantes de sua resposanbilidade em seu território. (BRASIL, 2012)

\subsection{CAPÍTULO 3 - Papel do enfermeiro no acompanhamento de pré-natal de baixo risco utilizando a abordagem centrada na pessoa}

O período gestacional único na vida de uma mulher, com grandes alterações em toda a vida da mesma (físicas, psíquicas, emocionais e em todo contexto social e familiar). Cuidar da gestante à construção de estratégias de atenção à saúde materna devido a todas essas alterações.

No que se refere à atuação do enfermeiro frente às ações de assistência integral à saúde da mulher, considera-se que o enfermeiro em sua formação acadêmica está habilitado para realizar a consulta de enfermagem e a assistência ao pré-natal de baixo risco. Além disso, esse procedimento é respaldado em lei (em respeito à Lei do exercício profissional 7499/86 e o Decreto 94.406.187 e portaria 1721/MEC de 15/12/1994) que confere ao enfermeiro a habilitação necessária para o exercício desta função (BRASIL, 2004)

De acordo com a organização e regulamentação das ações do profissional enfermeiro, há algumas décadas as Secretarias Municipais de Saúde dos municípios 
brasileiros protagonizam protocolos de acordo com as referências teóricas e necessidades/demandas de cada população (BRASIL, 2006)

A atenção primária à saúde das gestantes inclui as condutas de prevenção de doenças e agravos, a promoção da saúde materno-fetal e o tratamento dos problemas ocorridos durante o período gestacional. O Ministério da Saúde objetiva que a atenção às gestantes deve reduzir as taxas de morbimortalidade materno-infantil, à proporção em que são adotadas medidas satisfatórias do acompanhamento ao pré-natal (BRASIL, 2006)

Segundo Osava \& Tanaka (1997), em termos históricos, a enfermagem sempre esteve presente no acompanhamento e avaliação de mulheres em período gestacional, vista que a enfermeira exerce papel fundamental na realização de parto e vem recebendo várias designações no decorrer dos anos como parteira, obstetriz e enfermeira obstetra.

Lima e Moura (2005), reforçam que a consulta de enfermagem é uma atividade que concede à família, ao indivíduo ou à comunidade o direito a promoção da saúde seja tanto no âmbito hospitalar quanto no domiciliar.

A consulta de enfermagem é um conjunto de ações privativas ao enfermeiro que foca o saber e o fazer, compreendendo o cuidado do ser humano e suas particularidades (MARQUES e PRADO, 2004)

No que se refere à atuação do enfermeiro frente às ações de assistência integral à saúde da mulher, considera-se que o enfermeiro em sua formação acadêmica está habilitado para realizar a consulta de enfermagem e a assistência ao pré-natal de baixo risco. Além disso, esse procedimento é respaldado em lei (em respeito à Lei do exercício profissional 7499/86 e o Decreto 94.406 .187 e portaria 1721/MEC de 15/12/1994) que confere ao enfermeiro a habilitação necessária para o exercício desta função (BRASIL, 2004)

$\mathrm{O}$ enfermeiro que atua em uma ESF tem que exercer um papel humanizador junto às gestantes, porque é na primeira consulta que começa o contato entre $o$ enfermeiro e a gestante formando um vínculo de confiança. Um pré-natal de qualidade é o principal caminho para um bom parto (LIMA e MOURA, 2005).

Mas, uma assistência de qualidade não necessariamente depende de procedimentos só técnicos de alta complexidade, pois uma das qualidades desta assistência consiste em um relacionamento de confiança entre o profissional e a gestante (SANTOS, 2012).

Nesse contexto com uso de tecnologias leves, utilizadas pelo próprio profissional no relacionar-se com a gestante durante a consulta de Enfermagem que reforço o uso da abordagem centrada na pessoa, centrada na gestante foco principal do pré-natal. Pelo 
cuidado com a mesma e com seu bebê. Ao cuidar bem da mulher, diretamente e indiretamente estamos trazendo um cuidado integral, resolutivo, com equidade e todos os princípios que integram o SUS e podem ser sempre utilizados na ESF.

$\mathrm{O}$ essencial para a equipe que trabalha com as gestantes, principalmente enfermeiros é saber compreender o que está acontecendo com a grávida, pois por trás de cada pergunta, por mais ingênua que seja, poderão existir importantes questões emocionais e ocultas que podem trazer impacto na saúde do Binômio mãe bebê (LIMA e MOURA, 2005).

A assistência ao pré-natal deve proporcionar diálogos que tratem a mulher como um ser integral, num contexto familiar, social, sem expor sua individualidade, emoções e dificuldades externas. Com a valorização do contexto familiar juntamente com enfoque na saúde da mulher, passa a existir uma unidade de ação programática de saúde, buscando parcerias garantindo a eficácia das ações e demandas das gestantes, bem como desenvolver ações preventivas na educação em saúde (BRASIL, 2012).

A Consulta de Enfermagem no pré-natal engloba as atividades de: anamnese, exame físico, solicitação e/ou interpretação de exames laboratoriais e orientação. Destaca-se que, quanto à orientação, o enfermeiro aborda temáticas como aleitamento materno, alimentação e pré-natal, dentre outras. Ainda durante a consulta, deve-se propor e ajudar a prevenir o desenvolvimento de agravos comuns durante a gravidez e favorecer a vivência de uma gestação tranquila, na qual a mulher sinta-se segura, tendo um bom parto (NERY; TOCANTINS, 2006).

Para Marques e Prado (2004) é atribuída à enfermeira, durante a consulta de enfermagem no pré-natal, a tarefa de orientar as mulheres e suas famílias sobre a importância da realização contínua deste, da amamentação, da vacinação, do preparo para o parto. Ainda, a enfermeira tem a tarefa de proporcionar um acolhimento adequado à gestante através de uma boa interação, conversando, ouvindo com interesse, valorizando atitudes ou ações condizentes à saúde e envolvendo o parceiro e a família. Como se percebe através dos estudos elencados, a consulta de enfermagem contribui para a melhoria da qualidade de vida da gestante, assim como, para o vínculo entre profissional e cliente, e isso é imprescindível para uma assistência otimizada. Dessa forma, a Consulta de Enfermagem proporciona orientação de medidas favoráveis, que visam à abordagem apropriada às necessidades peculiares de cada mulher com as quais interagimos em consultas no pré-natal, nas unidades básicas de saúde. (LIMA; MOURA, 2005).

O profissional enfermeiro pode acompanhar inteiramente o pré-natal de baixo risco na rede básica de saúde, de acordo com o Ministério de Saúde e conforme 
garantido pela Lei do Exercício Profissional, regulamentada pelo Decreto n ${ }^{0}$ 94.406/87. (BRASIL, 2012)

O Ministério da Saúde (MS), estabeleceu um mínimo de seis consultasde prénatal, durante todo o período que antecede o nascimento. Os aspectos emocionais devem ser abordados desde a primeira consulta de prénatal, estabelecendo uma confiança entre o profissional e a gestante, assim mais dúvidas podem ser sanadas e há uma abertura maior por parte da gestante (BRASIL, 2006).

Durante a consulta de enfermagem, além da competência técnica, o enfermeiro deve demonstrar interesse pela gestante e pelo seu modo de vida, ouvindo suas queixas e considerando suas preocupações e angústias. Para isso, o enfermeiro deve fazer uso de uma escuta qualificada, a fim de proporcionar a criação de vínculo. Assim, ele poderá contribuir para a produção de mudanças concretas e saudáveis nas atitudes da gestante, de sua família e comunidade, exercendo assim papel educativo. (BRASIL, 2012)

$\mathrm{Na}$ instância do cuidado pré-natal pelo enfermeiro é necessário valorizar os sentimentos e experiências relacionados à gravidez. $O$ ouvir atentamente sem julgamentos, o respeito, a empatia, a tolerância, a disponibilidade, a confiança, o diálogo, a preservação da individualidade do outro e a troca de experiências fazem-se necessários, a fim de que o cuidado possa repercutir não só na qualidade dos sentimentos manifestados pela mulher, mas também culminar em uma adequação saudável da gestante ao seu papel materno.

A Consulta de Enfermagem no pré-natal engloba as atividades de: anamnese, exame físico, solicitação e/ou interpretação de exames laboratoriais e orientação. Destaca-se que, quanto à orientação, o enfermeiro aborda temáticas como aleitamento materno, alimentação e pré-natal, dentre outras. Ainda durante a consulta, deve-se propor e ajudar a prevenir o desenvolvimento de agravos comuns durante a gravidez e favorecer a vivência de uma gestação tranquila, na qual a mulher sinta-se segura, tendo um bom parto (NERY e TOCANTINS, 2006).

Para Marques e Prado (2004) é atribuída à enfermeira, durante a consulta de enfermagem no pré-natal, a tarefa de orientar as mulheres e suas famílias sobre a importância da realização contínua deste, da amamentação, da vacinação, do preparo para o parto. Ainda, a enfermeira tem a tarefa de proporcionar um acolhimento adequado à gestante através de uma boa interação, conversando, ouvindo com interesse, valorizando atitudes ou ações condizentes à saúde e envolvendo o parceiro e a família. Como se percebe através dos estudos elencados, a consulta de enfermagem contribui para a melhoria da qualidade de vida da gestante, assim como, para o vínculo entre profissional e cliente, e isso é imprescindível para uma assistência otimizada. Dessa 
forma, a Consulta de Enfermagem proporciona orientação de medidas favoráveis, que visam à abordagem apropriada às necessidades peculiares de cada mulher com as quais interagimos em consultas no pré-natal, nas unidades básicas de saúde. (LIMA; MOURA, 2005).

Todas essas estratégias, ferramentas citadas acima fazem parte da abordagem centrada na pessoa. Como foi amplamente citado no capiulo anterior por Ceron (2012).

Segundo Marques (2004) preconiza que na consulta de enfermagem às gestantes, deve ocorrer à participação ativa da cliente através da interação com o profissional enfermeiro, em que ambos trocam saberes e informações visando à promoção do auto cuidado. Nessa perspectiva, através da consulta de enfermagem como um momento de diálogo, entre enfermeiro/cliente pode-se definir metas e objetivos a serem atingidos visando à melhoria nas condições de saúde do binômio mãe e filho.

Moira (2010) reforça que com o uso da abordagem centrada na pessoa (ACP), a ampliação do cuidado, o profissional promove uma relação de cooperação com o paciente. ambos encontram-se num terreno comum para abordar as preocupações da gestante, e as decisões a serem tomadas e as suas ideias sobre o que ocorre e o que deve ser feito, leva-se em conta as suas expectativas e as suas experiências pessoais e culturais em relação à gestação, e também as de sua comunidade e família.

$\mathrm{O}$ enfermeiro precisa entender que a comunicação dialógica deve ser fundamentada na prática do cuidar, e não fazer tentativas de controlar ou modificar a pessoa ou prescrever somente tratamentos; mas sim, estar disposto a interagir, ensinar e aprender com o indivíduo e com o coletivo, através de ações educativas (RIO DE JANEIRO, 2016).

Desta forma, a Consulta de Enfermagem no pré-natal, proporciona a aplicação de medidas favoráveis que visam à abordagem apropriada às necessidades peculiares das mulheres, visando o monitoramento do bem - estar da gestante, o desenvolvimento do feto e a detecção precoce de quaisquer problemas. (LIMA, 2005)

$\mathrm{O}$ enfermeiro realiza as consultas de enfermagem no pré-natal, sendo estabelecido pelo Ministério da Saúde (MS) o mínimo de seis consultas durante todo o período que antecede o nascimento. Os aspectos emocionais devem ser abordados desde a primeira consulta de prénatal, estabelecendo uma confiança entre o profissional e a gestante, assim mais dúvidas podem ser sanadas e há uma abertura maior por parte da gestante (BRASIL, 2006).

A assistência no pré-natal, adicionada ao cuidado inclui a qualidade do enfermeiro, que deve ser competente, humano e dedicado. Competente para orientar convenientemente as gestantes, reconhecer precocemente os problemas que possam 
surgir e enfrentá-los de maneira correta de modo a evitar ou minimizar suas consequências (FREITAS, 2007).

Assim, a atenção à saúde da mulher deve ser organizada de forma que possa atender as reais necessidades das mulheres durante a gestação e o puerpério, mediante a utilização dos conhecimentos técnico-científicos existentes e dos meios e recursos (humanos e físicos) disponíveis adequados para cada caso garantindo a humanização da assistência e dessa forma reduzindo a morbimortalidade materna e infantil (BRASIL, 2006).

A saúde da mãe é um determinante importante durante o período gestacional. É dela que depende o bem-estar do seu futuro filho. Por esse motivo, faz-se necessário que durante a gestação a mulher seja orientada e encorajada a buscar medidas que previnam qualquer intercorrência que possa prejudicar o crescimento e o desenvolvimento do bebê (BEZERRA, 2009).

A assistência pré-natal é de fundamental importância para preparar a mulher para a maternidade, não devendo ser encarada como simples assistência médica e sim, como trabalho de prevenção de intercorrências clínico-obstétricas e assistência emocional (BRASIL 2006).

Para o Ministério da Saúde (2006), as gestantes estão buscando o pré-natal devido à qualidade da assistência prestada pelo serviço e profissionais de saúde, uma vez que elas estão cada dia mais consciente da importância deste atendimento para a diminuição dos elevados índices de mortalidade materno-fetal.

Diante disso é importante ressaltar que o SUS preconiza dentre seus princípios e diretrizes a integralidade da assistência que é entendida como conjunto articulado e continuo das ações, serviços preventivos e curativos, individuais e coletivos, exigido para cada caso em todos os níveis de complexidade do sistema, e a integralidade na assistência depende primordialmente de quem a pratica (BRASIL, 2006).

Desta forma vai depender do enfermeiro que a consulta tenha nuances diferentes, para atender as expectativas da paciente, fazendo com que aconteça uma relação de confiança e troca entre o profissional e paciente, preconizando uma assistência de qualidade humanitária. (RIOS e VIEIRA, 2007)

Atualmente, a consulta de enfermagem na rede básica de saúde é realizada de acordo com roteiro estabelecido pelo Ministério da Saúde. Este trabalho se justifica pelo fato do pré-natal ser o acompanhamento e acolhimento que a gestante recebe, a fim de proporcionar promoção, prevenção e tratamento de possíveis intercorrências durante o período gestacional e pós-parto, além de preparar a gestante para um puerpério saudável e tranqüilo. (RIOS e VIEIRA, 2007) 
O enfermeiro desempenha um papel de extrema importância, para que ocorra um pré-natal de qualidade já que a equipe de enfermagem deve estar apta a realizar uma assistência humanizada, baseada na atenção às queixas da paciente, executando e prescrevendo cuidados, orientações de qualidade durante o atendimento. Na consulta de pré-natal o enfermeiro e sua equipe desenvolvem assistência integral a gestante por meio de ações e procedimentos técnicos e científicos, assegurando uma gestação sem intercorrências ou minimizando os agravos/desconfortos que podem surgir no decorrer da gestação (BEZERRA, 2009).

Durante a gestação a mulher passa por mudanças anatômicas, fisiológicas e psicológicas. O pré-natal é um momento em que a mulher tem para conhecer e compreender as mudanças que estão ocorrendo em seu corpo, à equipe de enfermagem utilizando- se de ações e atividades educativas como forma de sanar dúvidas em relação a uma alimentação saudável, preparar as gestantes para realização de cuidados com o recém-nascido, e a importância do aleitamento materno, promove dessa maneira uma gestação e puerpério tranqüilo (BEZERRA, 2009).

Contudo a equipe de enfermagem da rede básica de saúde necessita estar apta com conhecimentos técnicos, científicos e humanizados para trabalhar. As gestantes, seus parceiros e suas famílias devem sempre ser tratados com gentileza, respeito e dignidade (RIO DE JANEIRO, 2016).

A Secretaria Municipal de Saúde do Rio de Janeiro (2016) recomenda o cuidado centrado na pessoa deve ser utilizado como ferramenta fundamental da Atenção Primária à Saúde (APS).

As mulheres devem ter a oportunidade de tomar decisões informadas sobre seu acompanhamento e tratamento, em parceria com os profissionais de saúde. Aceitar ou rejeitar os procedimentos propostos são direitos da usuária. Uma boa comunicação entre os profissionais e a gestante é essencial. Forneça informações que: sejam de fácil compreensão para todas as mulheres, incluindo aquelas com necessidades especiais; possibilitem às mulheres a tomada de decisões informadas e que sejam consistentes e baseadas nas melhores evidências disponíveis Recomenda-se o uso de material escrito para reforço destas informações. As informações devem contemplar: onde e por quem a gestante será acompanhada; o número e a periodicidade usual de consultas; e o convite para participação em grupos educativos sobre gestação e amamentação, se disponíveis (RIO DE JANEIRO, 2016).

Essas atitudes são fundamentais para uma atenção pré-natal adequada, que respeita as decisões da mulher e da sua família, mesmo quando as opiniões são contrárias às suas próprias; é essencial que o enfermeiro venha a prover oportunidades para que sejam discutidas preocupações e dúvidas dentro da consulta de pré-natal ou 
fora dela nos tanos espaços que a ESF propicia ao cuidado - salas de espera, grupos educativos, visita domiciliar, sala de vacinas (RIO DE JANEIRO, 2016)

O cuidado centrado na pessoa, na gestante, necessita também que seja avaliada se as informações pactuadas foram entendidas sempre oferecer tempo suficiente para a tomada de decisões, fornecer explicações sobre os exames indicados, propiciando discussão sobre o assunto. E todas as outras dúvidas apresentadas pela gestante, sempre valorizando as demandas trazidas pela mesma. Seja em uma comunicação v erbal ou não verbal. As mulheres devem sentir-se confortáveis para discutir questões íntimas e revelar problemas, num espaço seguro garantido durante o pré-natal, livre de qualquer julgamento (RIO DE JANEIRO, 2016).

\section{CONSIDERAÇÕES FINAIS}

O pré-natal é um acompanhamento da evolução da gestação que visa cuidar da saúde da mulher e do seu bebê até que o parto ocorra, também é o momento que a gestante vivencia diferentes sentimentos, por isso o estabelecimento de relação com a enfermagem se faz imprescindível.

Os resultados da revisão de literatura mostraram a importância da atuação dos profissionais enfermeiros junto às gestantes e famílias durante o período pré-natal. E que, quando o mesmo é realizado com qualidade desempenha importante papel na redução da mortalidade materna e infantil, um cuidado integral á gestante e o fortalecimento do vínculo, que propicia a manutenção do cuidado longitudinal que permanece ao fim, quando ocorre o nascimento do bebê.

A consulta de enfermagem é uma atividade que irá proporcionar ao enfermeiro condições para atuar de forma direta e independente com a paciente, caracterizando dessa forma sua autonomia. Segundo o Ministério da Saúde a humanização do pré-natal busca garantir a assistência à saúde da gestante, promovendo uma boa qualidade no atendimento e prevenção de possíveis complicações durante a gestação.

A boa qualidade desse acompanhamento pode ser atribuída ao uso da abordagem centrada na pessoa, durante o pré-natal, abordagem centrada na gestante foco da atenção do profissional junto com todas as demandas de cuidado trazidas por ela e sua família.

O Cuidado Centrado na Pessoa: aponta para o desenvolvimento de ações de cuidado de forma singularizada, que auxilie as pessoas a desenvolverem os conhecimentos, aptidões, competências e a confiança necessária para gerir e tomar decisões embasadas sobre sua própria saúde e seu cuidado de saúde de forma mais efetiva. O cuidado é construído com as pessoas, de acordo com suas necessidades e potencialidades na busca de uma vida independente e plena. A família, a comunidade e 
outras formas de coletividade são elementos relevantes, muitas vezes condicionantes ou determinantes na vida das pessoas e, por consequência, no cuidado

A grande expansão da Estratégia de Saúde da família, o incentivo para um cuidado humanizado favorece ao uso de Abordagem centrada na pessoa - na gestante e promove o fortalecimento como modelo vigente na Atenção primária, conforme exemplificado durante o trabalho através da PNAB.

Verificou-se o destaque deste profissional nas consultas, atendimentos individuais ou em grupos onde são abordadas questões referentes às relações familiares, cuidados com o recém-nascido, importância do acompanhamento pré-natal e a realização de todos os exames.

Como descrito na Lei n. ${ }^{\circ} 7.498$ de 25 de julho de 1986, que dispõe sobre a regulamentação do exercício de Enfermagem, diz que cabe à(o) enfermeira(o) realizar consulta de enfermagem e prescrição da assistência de enfermagem; como integrante da equipe de saúde: prescrever medicamentos, desde que estabelecidos em Programas de Saúde Pública e em rotina aprovada pela instituição de saúde; oferecer assistência de enfermagem à gestante, parturiente e puérpera e realizar atividades de educação em saúde.

Atualmente, a Consulta de Enfermagem na rede básica de saúde é realizada de acordo com o roteiro estabelecido pelo Ministério de Saúde (2000), garantida pela Lei do Exercício Profissional e o Decreto no 94.406/87, o pré-natal de baixo risco pode ser inteiramente acompanhado pelo enfermeiro. Nas atividades relacionadas à assistência pré-natal, estudos demonstrados por pesquisadoras relatam que a Consulta de Enfermagem tem sofrido transformações em sua concepção, metodologia e, principalmente, a inserção nos serviços de saúde, transitando para o prestígio e aceitação do profissional enfermeiro no seu fazer e assistir. Dessa forma, a consulta de enfermagem proporciona orientação de medidas favoráveis que visam à abordagem apropriada às necessidades peculiares das mulheres, das gestantes, com quem interagimos em consultas no pré-natal, nas unidades básicas de saúde.

É pertinente lembrar que os contatos frequentes nas consultas entre enfermeiros e gestantes possibilitam melhor monitoramento do bem-estar da gestante, o desenvolvimento do feto e a detecção precoce de quaisquer problemas. Assim, a consulta de enfermagem juntamente com atividades educativas podem preencher, muitas vezes, as lacunas de conhecimento, tornando-se um espaço de discussão e orientação e cuidado integral.

È importante reforçar que a ação da enfermagem não pode ser desenvolvida isoladamente sem conexão exata, e sim permanentemente com todos os outros 
profissionais que participam no decorrer deste processo. Tornando-se necessária a adoção de medidas e procedimentos sabidamente benéficos para o acompanhamento durante este período, evitando na maioria dos casos, práticas desnecessárias, que embora frequentemente realizadas, não beneficiam a mulher nem o recém-nascido e que acarretam maiores riscos para ambos.

O objetivo traçado foi alcançado e comprovado por diversos autores citados no trabalho. Foi possível perceber que a abordagem centrada na pessoa, vai totalmente ao encontro de toda a diretriz proposta para o cuidado na Estratégia de saúde da família e que com o enfermeiro possui em sua formação acadêmica e profissional, muitos saberes para uso e implementação da ACP.

Ao aproximar as habilidades relacionais, das técnicas, conjuntamente com vínculo podemos aproximar sobre a convergência entre as políticas públicas de saúde no Brasil e a proposta da Abordagem Centrada na Pessoa (ACP). Esta se trata de uma proposta psicológica que se aproxima teoricamente das diretrizes propostas pelas políticas públicas nos serviços de saúde do Brasil, que se alinha ao princípios e diretrizes do SUS e de toda a abordagem realizada Estratégia de saúde da família.

\section{REFERÊNCIAS}

AQUINO, M. M. A. Gestação - Pré-natal, atividades físicas e alimentação e ganho de

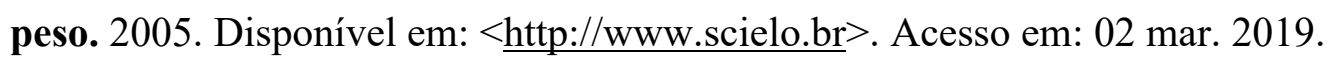

\section{BEZERRA, C. P. A importância da Consulta de Enfermagem no acompanhamento}

pré-natal. 2009. Disponível em: <http://www.fsm.edu.br/artigos/a-importancia-daconsulta-de-enfermagem-noacompanhamento-pre-natal/>. Acesso em: 14 fev. 2019.

BRASIL. Ministério da Saúde. Assistência Pré-Natal. Secretaria de Políticas de Saúde. Manual Técnico, $3^{\mathrm{a}}$ edição. Brasília, DF. Ministério da Saúde, 2000. 66 p. (Assistência Integral a Saúde da Mulher. Bases de Ação Programática, 60.).

BRASIL, Ministério da Saúde. Assistência pré-natal. Universidade Federal de Mato Grosso do Sul. 2000.

BRASIL. Ministério da Saúde. Política nacional de atenção integral à saúde da mulher: princípios e diretrizes. Brasília, DF. Ministério da Saúde, 2004. 
BRASIL. Ministério da Saúde. Pré-natal e puerpério: atenção qualificada e humanizada. Brasília, DF. Ministério da Saúde, 2006.

BRASIL. Secretaria de Atenção à Saúde. Departamento de Ações Programáticas Estratégicas. Saúde da Família e a Atenção Pré-Natal e Puerperal. Secretaria de Atenção à Saúde, ed. Rosa Reis, n. 36, ano VII, 2006.

BRASIL. Ministério da Saúde. Secretaria de Atenção à Saúde. Núcleo técnico da Política Nacional de Humanização. Acolhimento nas práticas de produção de saúde. $2^{\circ} \mathrm{ed}$. Brasília, DF: Ministério da Saúde, 2006. (Série B: Textos Básicos de Saúde).

BRASIL. Ministério da Saúde. Secretaria de atenção a saúde, Departamento de ações pragmáticas estratégicas. Política nacional de atenção integral a saúde da mulher: Princípios e diretrizes. Brasília, DF. Ministério da Saúde, 2009.

BRASIL. Ministério da Saúde. Secretaria de Atenção à Saúde. Departamento de Atenção Básica. Atenção ao pré-natal de baixo risco. Brasília, DF. Ministério da Saúde, 2012. 318 p. (Série A. Normas e Manuais Técnicos) (Cadernos de Atenção Básica, n 32).

BRASIL. Portaria $n^{\circ} 2.436$, de 21 de setembro de 2017. Aprova a Política Nacional de Atenção Básica, estabelecendo a revisão de diretrizes para a organização da Atenção Básica, no âmbito do Sistema Único de Saúde (SUS). Diário Oficial União. 22 set 2017; Edição: 183, Seção 1, p. 68.

BAULI, J. D. Avaliação da assistência pré-natal na rede básica do Município de Maringá - Paraná. [Dissertação de Mestrado em Enfermagem] Universidade Estadual de Maringá. Maringá, PR. 2010.

CERON, M. Habilidades de comunicação: abordagem centrada na pessoa. 2012. Disponível em $<$ http://www.unasus.unifesp.br/biblioteca_virtual/esf/2/unidades_conteudos/unidade24/uni dade24.pdf>. Acesso em: 25 abr. 2019.

FREITAS F., et al. Rotinas em obstetrícia. $5^{\circ}$ ed. Porto Alegre, RS: Artmed, 2007. 
LIMA, Y. M. S. MOURA, M. A. V. Consulta de Enfermagem pré-natal: a qualidade centrada na satisfação da cliente. R. de Pesq.: cuidado é fundamental. Rio de Janeiro, RJ. 2005. Ano 9, n. 1/2, p. 93-99. Disponível em: $<$ http://www.unirio.br/repef/arquivos/2005/10.pdf>. Acesso em 20 mar. 2019.

MAgalhães, D. R. B.; MAgalhães, E. B.; MOREIRA, A. B. C. Assistência PréNatal. In: CORREAA, M. D. et al. Noções práticas de obstetrícia. 13. ed. Belo Horizonte, MG: COOPMED Ed., 2004. p 69-89.

MARQUES, R. G. PRADO, S. R. L. A. Consulta de enfermagem no prénatal. Rev Enferm UNISA. 2004; v. 5, p. 33-6. Disponível em: $<$ http://www.unisa.br/graduacao/biologicas/enfer/revista/arquivos/2004-07.pdf. $>$ Acesso em: 03 abr. 2019.

MINAYO, M. C. S. O desafio do conhecimento: pesquisa qualitativa em saúde. $8^{\mathrm{a}}$ ed. São Paulo:Hucitec. 2004.

MOIRA, S. Medicina centrada na pessoa: transformando o método clínico [recurso eletrônico]; tradução: Anelise Burmeister, Sandra Maria Mallmann da Rosa; revisão técnica: José Mauro Ceratti Lopes. 3. ed. Porto Alegre, RS: Artmed, 2017.

NEME, B. Obstetricia básica. 2a edição. São Paulo: Sarvier. 2000. p. 118-120.

NERY, T. A.; TOCANTINS, F. R. O enfermeiro e a consulta pré-natal: o Significado da ação de assistir a gestante. Rev. enferm. UERJ Rio. 2006. v.14, n.1, p.87-92.

NETTO, H. C. Obstetrícia Básica. São Paulo, SP: Editora Atheneu, 2005.

OSAVA, R.H.; TANAKA, A. C. D. A. Os paradigmas da enfermagem obstétrica. Revista da Escola de Enfermagem da USP. 1997. v. 31, n. 1, p. 96-108.

RIBEIRO, Josiele Zorzolli Bretanha. Importância das orientações no pré-natal: conhecendo a visão das puérperas. 2011. 54f. Trabalho acadêmico - Graduação em Enfermagem. Universidade Federal de Pelotas, Pelotas - RS 
RIO DE JANEIRO, Secretaria Municipal de Saúde. Superintendencia de atenção primaria de saúde. Atenção ao Pre-natal. Rotina para gestantes de baixo risco. Rio de janeiro, 2016.

RIOS, C. T. F.; VIEIRA, N. F. C. Ações educativas no pré-natal: reflexão sobre a consulta de enfermagem como um espaço para educação em saúde. Ciência e Saúde Coletiva. 2007. v.12, n.2.

RIQUINHO DL, Correia SG. Mortalidade materna: perfil sócio-demográfico e causal.

Rev. bras. enferm. [Internet]. 2006 Disponível em: http://www.scielo.br/scielo.php?script=sci_arttext\&pid=S0034-71672006000300010 \&lng=en.Acesso em 02 de abril de 2019

SANTOS, D. S. et al. Sala de espera para gestantes: uma estratégia de educação em saúde. Revista brasileira de educação médica. 2012. v. 36, n. 1, p. 62-67.

TRENTINI, M. PAIM, L. Pesquisa em enfermagem: Uma abordagem convergenteassistencial. Florianópolis, SC: Editora da UFSC, 1999.

WOOD, J. K.; Doxsey, J. R.;ASSUMPÇÃO L. M.; TASSINARI, M. A.; JAPUR, M.; SERRA, M. A.; WRONA, R.; LOUREIRO, S.R.; CURY,V. E. (Orgs.). (2008). Abordagem Centrada na Pessoa. (4a ed.). Vitória: Edufes. 